CORONARY DISEASE

\section{Role of stenting in coronary}

revascularisation

Anthony H Gershlick

Glenfield Hospital, Leicester, UK angioplasty alone was the main method of undertaking PCI. A number of studies had demonstrated its superiority over medical treatment alone, but results varied when it was compared to coronary artery bypass surgery (CABG). The ACME trial compared angioplasty with medical treatment for patients with single vessel disease and exercise induced myocardial ischaemia. At six months $64 \%$ of the medically treated group still had angina compared to $46 \%(\mathrm{p}<0.01)$ of the angioplasty group who were largely not taking anti-anginal medication. ${ }^{\mathrm{w} 2}$ The value of intervention in improving symptoms was further supported by the RITA-2 trial, ${ }^{\text {w3 }}$ with a significant improvement in exercise tolerance in those treated with angioplasty. The longer term results versus surgery were, however, less convincing.

The RITA-1 trial data compared outcome in patients with single or multivessel disease considered suitable for angioplasty or coronary surgery who were then randomised to one or other treatment. The results ${ }^{\mathrm{w} 4}$ showed that early mortality was similar, but at six months those patients randomised to coronary angioplasty (PTCA) had a higher incidence of angina ( $32 \% v 11 \%)$, a greater need for repeat coronary angiography ( $31 \%$ v $7 \%$ for surgery), and a higher need for revascularisation $38 \% \mathrm{v}$ $11 \%$ ) compared to those patients who underwent surgery. This supported previous published observational data on the early natural history of angioplasty, which reported restenosis following PTCA in up to $40 \%$ of patients. ${ }^{\text {w5 }}$ This recurrence rate did not fall despite multiple drug trials designed to test whether the response of the vessel wall to balloon damage could be attenuated. ${ }^{\mathrm{w} 6}$ It had been established that restenosis, if it was going to happen, would occur within the first 4-6 months. The two year follow up data from the RITA trial supported this, when the angina rate for PTCA had not changed $(31 \%$ v $32 \%$ at six months), but it had risen in the surgical group from $11 \%$ to $22 \%$.

Despite the possible equalisation of outcome with time, PTCA alone remained an unsatisfactory treatment. Published metaanalyses $^{12}$ of other comparative trials indicate that while there is no notable difference in mortality between PTCA and surgery at one and three years, further intervention is required more frequently in the angioplasty patients; in the first year $33.7 \%$ of patients initially treated with angioplasty required a further procedure compared to $3.3 \%$ of those treated with surgery $(p=0.006)$ (fig 1$)$. While angina rates were higher early after angioplasty, by three years the incidence of angina was the same. It has been proposed that longer term results may favour non-surgical intervention since there is a $2.5 \%$ vein graft attrition rate per year with only $50 \%$ of grafts patent at 10 years. Up to $15 \%$ of angioplasty procedures are undertaken in patients who have had previous coronary artery bypass grafts. The results of balloon angioplasty alone were still unacceptable, however.
Correspondence to: Dr AH Gershlick, Glenfield Hospital, Leicester LE3 9QP, UK agershlick@aol.com

\section{Why stents?}

The development of stenting and its current position in PCI came about to some degree by accident. Until the mid 1990s balloon 


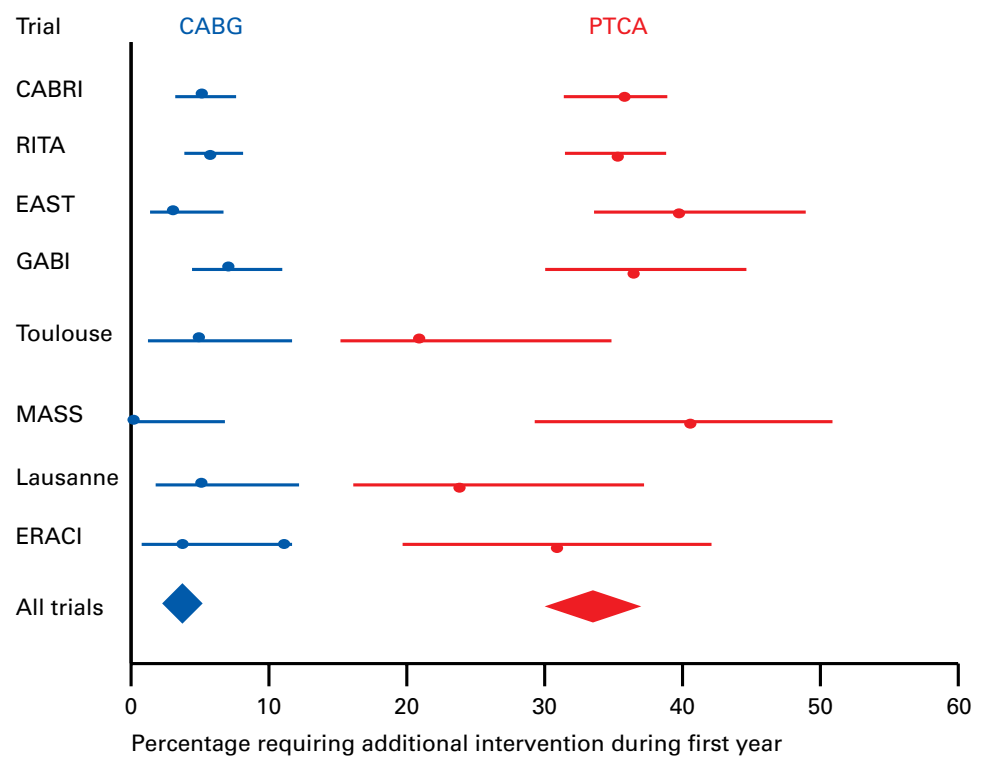

Figure 1. Trials of percutaneous transluminal coronary angioplasty (PTCA) versus coronary artery bypass graft (CABG) surgery. When angioplasty alone is used, the need for intervention during the first year is much greater than for surgical patients. which time stenting is more difficult and potentially unsuccessful. Two year survival following stenting for acute bailout has been reported to be $95 \%$, but event-free survival was only $70.7 \% .^{7}$ Recent changes in stent design, deployment techniques, and treatments to prevent thrombotic occlusion and restenosis in higher risk patients will, it is hoped, improve this longer term outcome, but it highlights the issue of restenosis.

- Consensus-Acute closure after balloon angioplasty carries a high complication rate.

Chronic problems: restenosis following angioplasty

Initial concepts on recurrence or restenosis after balloon angioplasty centred on the neointimal (smooth muscle cell) response. However, clinical drug trials to limit the impact of smooth muscle cell hyperplasia were generally unsuccessful. Concepts evolved to include the importance of the acute luminal diameter gain, recoil, and the impact of negative remodelling $^{8}$ (whereby the restenosing artery gets smaller rather than bigger to accommodate the intraluminal tissue) (fig 2). It was generally felt that such mechanical issues were more important than the impact of tissue in-growth. This meant that stent deployment could have an impact on recurrence since it would deal with three of the four factors thought to be important.

The larger the lumen that can be achieved and maintained after PCI, the less impact any restenotic tissue might have. ${ }^{9}$ Additionally $60 \%$ or so of the loss of lumen is caused by elastic recoil and negative remodelling. Quantitative angiographic data from a number of trials clearly showed that stents produced a significantly greater acute luminal gain, ${ }^{10}$ and prevented recoil. Although tissue response to stenting is exacerbated it has less impact since the arterial lumen is larger. Trial data ${ }^{10}$ indicate that the difference in final acute minimal luminal diameter can be increased from about $1.7 \mathrm{~mm}$ with angioplasty to about $2.7 \mathrm{~mm}$ (158\%) with stents.

- Consensus-Evolving concepts on restenosis suggest a mechanical solution is likely to reduce recurrence rates. the time of urgent surgical referral. Kioka and colleagues $^{4}$ reported an astonishing $15.8 \%$ mortality.

By the mid 1990s stent use was being advocated to improve outcome following acute coronary closure, the concept being that scaffolding of the loose and potentially obstructive intima would restore flow and reduce the need for emergency coronary surgery. While there are few large observation studies or any randomised trials, outcome following stent placement for bailout appeared good, with mortality rates of less than $5 \% .^{5}$ Roubin has published data on bailout stenting indicating an optimal angiographic result can be obtained in $93 \%$ of patients and a mortality rate of $1.7 \% .{ }^{6}$ Clinical evidence suggests that the earlier the stent is deployed after a poor angiographic result, the better the outcome. Indeed, one reason for the increase in stent use was the desire not to wait until the artery closed, at

Balloon intervention suffers two major prob"controlled" disruption of the atheromatous plaque, which sometimes becomes less control(hutished data suggest that outco than the outcome following stenting and worse authors ${ }^{3}$ have reported mortality rates of between $5-10 \%$ in such circumstances, de-

Is the use of stents justified?: clinical trials

Stenting versus angioplasty alone

Two major trials were designed to assess the medium term angiographic and clinical outcome following de novo (primary) stenting. Angioplasty in these trials was the preliminary procedure to stent delivery but could be used to optimise the post-stent lumen. The two trials (BENESTENT I and STRESS) have clearly shown that stenting in native vessels reduces the incidence of recurrence. In terms of reduction in restenosis rates, the results were remarkably similar in both trials. ${ }^{10}$ In the BENESTENT study, the primary clinical end points of myocardial infarction, need for 


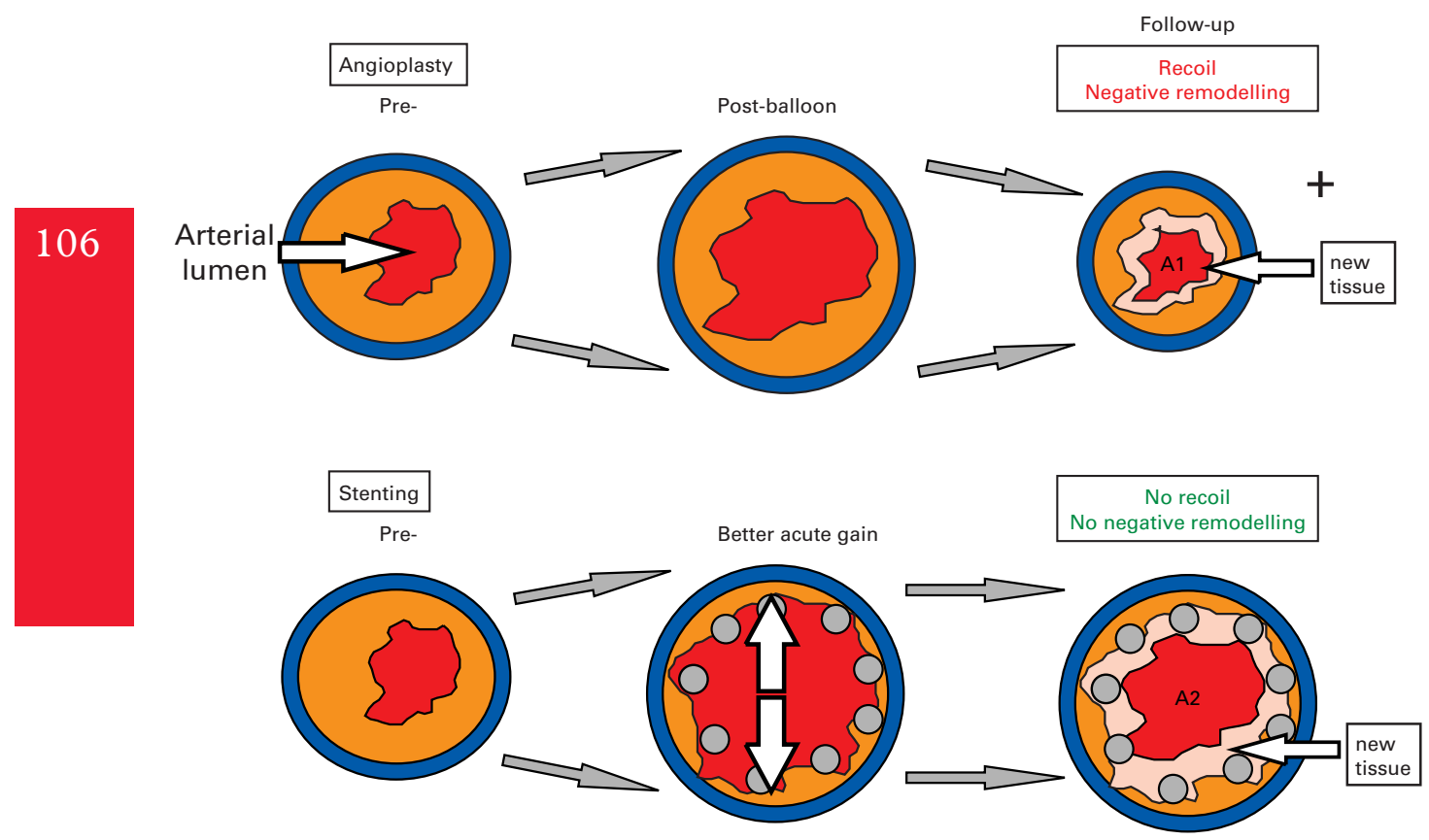

Figure 2. Stents improve outcome by improving the acute gain and by reducing recoil and negative remodelling, although tissue growth is not affected and may even be greater with stents than balloon angioplasty. Even so, the eventual lumen diameter is still larger after stenting (A2 > A1).

CABG or re-PTCA, and stroke had a relative risk of 0.68 (95\% confidence interval 0.5 to 0.92) in those patients randomised to stenting compared to those undergoing PTCA alone $(\mathrm{p}=0.02)$. The angiographic restenosis rate, measured quantitatively on follow up angiogram, was $22 \%$ for stenting and $32 \%$ for PTCA. For the STRESS trial ${ }^{11}$ the restenosis rate was $29.1 \%$ for stenting versus $42 \%$ in the PTCA arm ( $\mathrm{p}=0.011)$.

A number of equivalence studies have now been published comparing newer stents with the stents used in these trials. Any stent which produces a good acute result leads to recurrence rates of between 15-20\% compared to historical results of $35 \%$ for PTCA. ${ }^{12}$ The WEST, ${ }^{\text {w7 }}$ MUSIC, ${ }^{\text {w8 }}$ and FINESS ${ }^{\text {w9 }}$ trials have confirmed even lower restenosis rates. Use of intravascular ultrasound to optimise the best possible result leads to restenosis rates of $<10 \%$, but this cannot be justified in terms of time and costs.

\section{Effect of stent use on clinical practice}

While appropriately conducted clinical trials have shown a clear benefit of stenting compared to angioplasty, such trials are sometimes criticised for not being "real life" in that there is always some degree of patient selection. The case for stents in reducing the need for subsequent reintervention is supported further by indirect evidence from the British Cardiovascular Intervention Society (BCIS) audit data. From 1992 to 1998 there was an increase in angioplasties from 11575 to 24661 (113\%) with an increase in case complexity. During the same period there was a reduction in reintervention rates for restenosis (from $11.6 \%$ to $5.2 \%$ ) mirrored by an increase in stent use from $2.7 \%$ of all cases to $69 \%$. This suggests that an increase in stenting reduces the clinical need for reintervention (table 1).
Is there too much stenting?

It has been suggested that stenting is used uncritically and unnecessarily. ${ }^{13}$ A study designed to assess the potential added value of stenting once a good angioplasty result had been obtained has been published. ${ }^{\text {w10 }}$ In this trial (involving only 116 patients) if residual stenosis was less than $0.3 \mathrm{~mm}$, patients were randomised to either stent or no stent; $13.5 \%$ of patients developed recoil at 30 minutes and crossed over to the stent group. The results showed that there was no difference in restenosis rate, event-free survival, and target lesion revascularisation between the two groups at follow up. There are a number of important issues related to this study. Firstly, interventionists do not generally stent when they achieve a stent-like result. There are certain other messages, however. Of 953 angioplasties considered for this trial only $116(12 \%)$ met the criteria for the study, suggesting that achieving a "stent-like" result in everyday practice is difficult. It is unclear from the cost benefit analysis whether the excess number of balloons that are frequently required to achieve a stent-like result have been included. Finally, one of the stents that was used predominantly

Table 1 Coronary stenting: impact on requirement for emergency $C A B G$ and the need for reintervention in the UK 1992-98

\begin{tabular}{llcll}
\hline Year & $\begin{array}{l}\text { Number of } \\
\text { PCIs }\end{array}$ & $\begin{array}{l}\text { Stent rate } \\
(\%)\end{array}$ & $\begin{array}{l}\text { Emergency } \\
\text { CABG rate } \\
(\%)\end{array}$ & $\begin{array}{l}\text { PCI for } \\
\text { restenosis } \\
(\%)\end{array}$ \\
\hline 1992 & 11575 & 2.7 & 2.0 & 11.6 \\
1993 & 12937 & 5.6 & 2.0 & 12.3 \\
1994 & 14624 & 13.5 & 1.8 & 11.4 \\
1995 & 17344 & 27.6 & 1.9 & 9.6 \\
1996 & 20511 & 45.9 & 1.5 & 9.4 \\
1997 & 22902 & 60.0 & 1.1 & 7.4 \\
1998 & 24661 & 69.0 & 0.6 & 5.2
\end{tabular}

CABG, coronary artery bypass graft; PCI, percutaneous coronary intervention. 
was the GR II stent, which is a coil stent and as such has been associated with a higher restenosis rates. Certainly for the lesions included, stent restenosis rates would be expected to be lower than that cited in this study.

It is clear that if a "stent-like" result can be achieved with one or two balloons then stenting (which with contemporary units requires only one predilatation balloon) need not be necessary. Such immediate success with angioplasty alone is, however, uncommon and attempts to obtain the $+5 \%$ to $-5 \%$ residual stenosis routinely seen after stenting is not without attendant risks of dissection and acute vessel closure, since larger balloons and higher pressure may be needed.

- Consensus-Stenting improves outcome compared to balloon angioplasty alone. Achieving a stent-like result with angioplasty alone is difficult.

\section{Stents versus surgery in 2001}

Previous trials of angioplasty versus surgery quoted were in the pre-stent era. Stents should have made an impact, reducing or negating the difference between PCI and coronary surgery. There are a number of randomised studies comparing stenting with surgery for multivessel disease.

The one year results of the ARTS trial ( $\mathrm{n}=1200$ ), which compared stenting (mean (SD) $2.7(0.2)$ stents per patient) to surgery (2.8 (1.1) anastomoses per patient) in multivessel disease have been published. ${ }^{14}$ Ninety three per cent of patients received an arterial graft, and the percentage incidence of patients with unstable angina was similar in each group (37\% and $36 \%$, respectively). The average duration of the procedures was 1.5 hours for stenting and 4 hours for surgery. The inhospital stays were 3.4 days and 11.3 days, respectively.

Treatment according to randomisation was successful in $97 \%$ of patients treated with stents and $96 \%$ of those treated with surgery. Only $0.5 \%$ of stented patients needed urgent

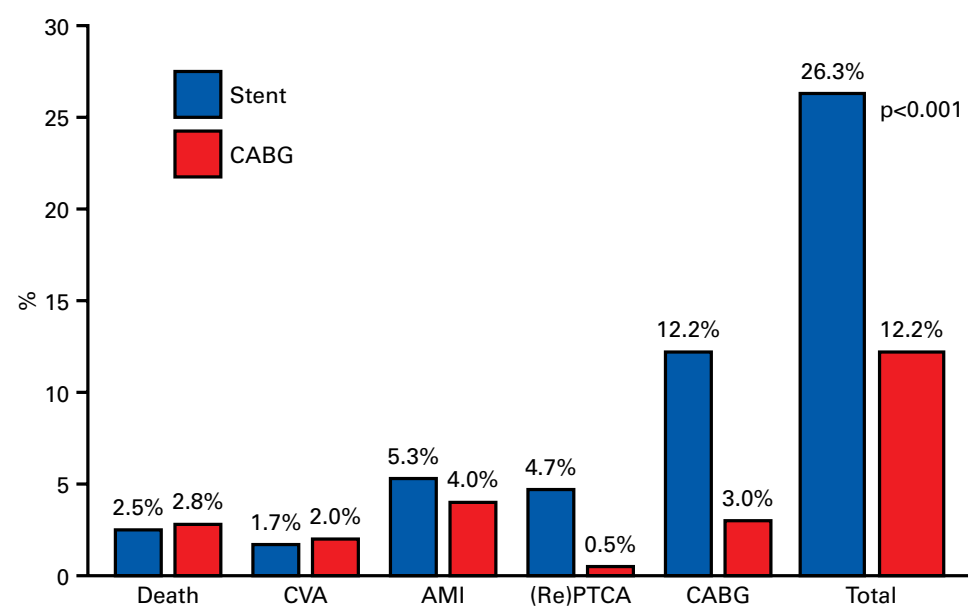

Figure 3. Residual problems with stenting relate to in-stent restenosis requiring repeat angioplasty (RePTCA) or bypass surgery (CABG). CVA, cerebrovascular accident; AMI, acute myocardial infarction. bypass grafting and a further $1.7 \%$ needed elective surgery. At one year the rates of death, acute myocardial infarction or stroke were low and did not differ between the groups $(9.5 \%$ for stented patients and $8.8 \%$ for surgical patients).

However, the event-free survival was higher in the surgical patients $(87.3 \% \quad v \quad 73.3 \%)$ entirely because of the need for reintervention in the stented patients (fig 3). While eliminating in-stent restenosis is the current research aim for many groups worldwide, even when as in this study it results in a $14 \%$ difference in need for reintervention, stenting remains cost effective compared to surgery. In the ARTS study stenting saved 4278 Euro in initial procedure costs compared to surgery, and although part of these savings were lost because of a higher need for revascularisation, the net savings at one year were 2965 Euro. ${ }^{\text {w1 }}$

The UK based SOS trial is shortly to be published in full.

Comparisons between angioplasty plus stenting and minimally invasive surgery to the left anterior descending artery are underway. Others such as the AMIST study have now had substantial funding approval and are recruiting.

- Consensus-In-stent restenosis caused by intimal hyperplasia remains a problem.

\section{Problems with stenting}

One problem with stenting is the residual incidence of restenosis. While this may be $<10 \%$ for intravascular ultrasound (IVUS) direct selected cases and $15 \%$ for trial BENESTENT lesions, even if these were real life the impact on the need for repeat procedures would be high. For the 1.2 million angioplasties carried out worldwide each year at a stent rate of $85 \%$, repeat procedures would be required in 153000 patients. The situation, however, is worse than this. Certain patient subsets have a higher incidence of in-stent restenosis. Data suggest that the rates are higher for both small vessels stented (up to $45 \%)^{15}$ and for multiple stents $(32 \%) .{ }^{16}$ Diabetics are a particular at risk group, and pre-stenting surgery was advocated based on the BARI trial data. In-stent restenosis rates of up to $55 \%$ have been quoted. ${ }^{17}$

\section{Dealing with in-stent restenosis}

In-stent restenosis remains one of the challenges for investigators. There is no doubt that treatment in the form of vascular brachytherapy is available. In-stent restenosis rates are reduced by about $60 \%$ and the clinical (target revascularisation) rates by about $50 \%$ irrespective of whether this is delivered as a $\beta$ emitter or a $\gamma$ emitter. ${ }^{\mathrm{w} 12-15}$ Two recently published trials (START $^{18}$ and INHIBIT ${ }^{19}$ ) specifically demonstrated that compared to placebo the use of ${ }^{90} \mathrm{Sr} / \mathrm{Y}$ and ${ }^{32} \mathrm{P}$ radionucleotide post reballooning to deliver $18 \mathrm{~Gy}$ at $2 \mathrm{~mm}$ from the source wall reduced the in-stent recurrence from 
$42.2 \%$ to $14.2 \%(\mathrm{p}<0.001)$ and from $48 \%$ to $16 \%$ ( $\mathrm{p}<0.0001)$, respectively. The incidence of tissue re-growth is greater at the edges of the stent $(28.8 \%$ and $26 \%$, respectively) which, although less than for placebo $(45.2 \%$ and $52 \%$, respectively), highlights one of the problems with the use of vascular brachytherapy. Others include the worry about long term outcome and in-stent thrombosis. The main problem, however, is that vascular brachytherapy is secondary treatment, and patients have to have developed in-stent restenosis first. A better approach would be primary prevention.

There is no doubt that it is the aim of all clinical investigators to eliminate or significantly reduce the incidence of in-stent restenosis, if only to reduce further the gap between PCI and surgery (currently around 14\% $\left.(\text { ARTS })^{14}\right)$. Local delivery of an anti-restenotic drug with the stent has the major advantage of being local and potentially cost effective. Two agents are currently in clinical trial.

Paclitaxel, an extract of the yew tree, has clinical use in ovarian cancer. It has specific properties with regard to microtubules, promoting polymerisation of tubulin. It inhibits the disassembly of microtubules, which thus become very stable and dysfunctional, so inhibiting cell division. ${ }^{\text {w16 }}$ We have now been able to coat stents with paclitaxel. In the work undertaken at John Hopkins using the pig coronary stent model, the inhibitory effects on smooth muscle activity appear to be dose related with an inhibitory, but not necessary linear, response at a dose of between $10 \mu \mathrm{g}$ and $187 \mu \mathrm{g}$ per stent. This, together with the dose related inhibitory effects on post-traumatic endothelial cell regeneration, have led at this stage to the initiation of a European pilot safety study (ELUTES). The trial completed recruitment in April 2001.

A large European based trial of sirolimus is also almost completed. Sirolimus is a naturally occurring macrocyclic lactone. It has been used as an immunosupressive agent in renal and islet grafting and for bone marrow transplantation. It binds to a specific cytosolic protein (immunophilin) found in target cells. This complex then binds to a specific regulatory kinase called the "mammalian target of rapamycin" (mTOR), inhibiting its activation, which in turn through inhibition of cell cycle progression suppresses cytokine stimulated $\mathrm{T}$ cell proliferation. However, it has other effects, including inhibition of translation of cdk4/ cyclin $\mathrm{D}$ and cdk2/cyclin $\mathrm{E}$ complexes, that are perhaps of greater potential for inhibiting in-stent restenosis. The RAVEL study will be reporting mid 2001. Early results are exciting.

Paclitaxel is being studied in the QUANUM study, being delivered on a specially designed stent (as opposed to the other studies involving a routine stent).

- Consensus: Vascular brachytherapy is the treatment of choice for diffuse in-stent restenosis. Trials on the use of drug eluting stents are currently underway.

\section{Stenting in certain patient subgroups}

Stenting following previous CABG

Long waiting lists for first time surgery, the success of the procedure, and the lower mortality and morbidity associated with angioplasty compared to re-do operation makes non-surgical intervention an attractive method of treating post-CABG patients (about $15 \%$ of all PCI). Restenosis rates after angioplasty to vein grafts are known to be higher than in native vessels, however, although the reasons are unclear. Rates of up to $60 \%$ have been reported. ${ }^{\mathrm{w} 17}$ In some small observational studies the rate of restenosis following stenting has been shown to be less (range 14-34\%). ${ }^{\text {w18 }} \mathrm{A}$ further observational study by the PalmazSchatz study group ${ }^{\text {w19 }}$ suggests that the clinical outcome and restenosis rates for stented grafts are comparable to those for angioplasty undertaken on native vessels. In other words, the excess rates associated with angioplasty to vein grafts may be "normalised" by stenting. A recently published study (saphenous vein de novo trial) confirmed a better clinical outcome in that the end points of freedom from death, myocardial infarction, repeated bypass surgery or revascularisation were reached less frequently in stented patients $(73 \%$ v 58\%, $\mathrm{p}=0.03) .^{\mathrm{w} 20}$ One further challenge for the treatment of old grafts with stents is the incidence of no reflow, where atherosclerotic debris occludes the distal vessel. A recent trial $\left(\mathrm{SAFER}^{\mathrm{w} 21}\right)$ suggests that the outcome (in terms of enzyme defined myocardial infarction) is better if a distal protection device is used.

Stents for treating occluded vessels

There is good evidence that using stents in the setting of balloon angioplasty for chronic total occlusions improves the outcome. This was shown in the SICCO trial $^{20}$ which reported that $57 \%$ of 119 stented patients were angina-free compared to $24 \%$ treated with angioplasty alone $(\mathrm{p}<0.001)$. Angiographic restenosis occurred in $32 \%$ of stented patients compared to $74 \%$ of the PTCA group $(\mathrm{p}<0.001)$ and reocclusion occurred in $12 \%$ and $26 \%$, respectively $(p=0.058)$. Target lesion revascularisation within 300 days was also less frequent in the stented patients $(22 \% v 42 \%, \mathrm{p}=0.025)$. The TOSCA study ${ }^{\mathrm{w} 22}$ confirmed the value of stenting in chronic total occlusions. Stenting produced a $45 \%$ reduction in clinically driven target lesion revascularisation at six months $(15.4 \%$ v $8.4 \%, \mathrm{p}=0.03)$.

Stents in unstable angina.

Despite some evidence that lesions in patients with unstable angina are more thrombogenic and are therefore potentially at higher risk, intervention for acute coronary syndromes accounts for $40 \%$ of the angioplasty workload. Support for the use of stents in the setting of unstable angina is available in the literature. Marzocchi and colleagues have recently published their experience of stenting in the setting of unstable angina. ${ }^{\mathrm{w} 23}$ Of 266 consecutive 
patients stented either electively (24\%), for bailout $(11 \%)$ or for suboptimal angioplasty result $(65 \%)$, the overall 30 day mortality was $0.3 \%$ (one patient with cardiogenic shock), and the incidence of non-fatal myocardial infarction was $2.2 \%$ (six patients). At longer term follow up (mean (SD) 17.7 (9.4) months) the cardiac mortality was $0.4 \%$, the myocardial infarction rate was $1.5 \%$, and the target lesion revascularisation rate was $9.3 \%$. Again certain subgroups, such as patients with diabetes or those who had longer stents implanted, adversely influenced the outcome.

\section{Stents and acute myocardial infarction}

Thrombolysis remains the mainstay of treatment for acute infarction. Intervention in the form of primary angioplasty has been shown to be better than angioplasty in terms of patency and longer term follow up. ${ }^{\text {w24 }}{ }^{\text {w25 }}$ However, primary angioplasty has a major drawback. Most patients with acute infarcts present to district general hospitals with no interventional facilities. However, in those that do undergo PCI a number of studies have shown that the use of stents appears to confer significant advantage despite the potentially thrombogenic combination of the stent and disrupted thrombogenic atheromatous plaque. The GRAMI trial ${ }^{\mathrm{w} 26}$ randomised 104 patients to either balloon angioplasty alone or to balloon angioplasty followed by stent deployment. While procedural success was equally high in both groups $(94.2 \% v 98 \%$, respectively), in-hospital adverse events were significantly less in the stented group $(3.8 \% \vee 19.2 \%$, $\mathrm{p}<0.03$ ), and at late follow up event-free survival was $83 \%$ for the stented group versus $65 \%$ for the angioplasty group $(p<0.002)$. Other small observational studies appear to show that restenosis rates in patients stented during acute infarction are no higher than for routine stented patients. ${ }^{\mathrm{w} 27 \mathrm{w} 28}$

The use of angioplasty with stenting, if considered necessary, as compared to repeat thrombolytic or conservative treatment in patients with failed thrombolysis is being tested in the UK based REACT trial being funded by the British Heart Foundation. A similar trial (MERLIN) is comparing repeat thrombolysis and intervention.

\section{Adjunctive pharmacological treatment}

The use of adjunctive drug treatment was addressed in a previous article in this series. ${ }^{21}$ In summary, the FANATASTIC, STARS, ISAR, and most importantly the CLASSICS study showed that antiplatelet treatment following stenting should include ADP receptor blocking agents (clopidogrel loading dose $300 \mathrm{mg}$ and then $75 \mathrm{mg}$ per day for a month) as well as aspirin.

The use of the glycoprotein IIb/IIIa inhibitors is more controversial. The various trials have assessed the monoclonal antibody abciximab (c-7 E3 Fab, ReoPro) (EPIC, EPILOG, CAPTURE, EPISTENT trials ${ }^{\mathrm{w} 29-32}$ ) and the synthetic small molecules tirofiban (Aggrastat) (PRISM-PLUS, RESTORE ${ }^{\mathrm{w} 33}{ }^{\mathrm{w} 34}$ ), and eptifibatide (Integrilin) (IMPACT, PURSUIT ${ }^{\mathrm{w} 35 \mathrm{w} 36}$ ). There is no doubt that these agents have an impact on trial end points, in particular that of enzyme defined myocardial infarction, in those patients with acute coronary syndromes who are troponin T/I positive (CAPTURE ${ }^{\mathrm{w} 31}$ ), and are especially valuable in this regard in those going on to PCI (CAPTURE, ${ }^{\text {w31 }}$ PRISMPLUS, ${ }^{\mathrm{w} 33}$ PURSUIT $\left.{ }^{\mathrm{w} 36}\right)$. Patients at high risk undergoing intervention undoubtedly benefit irrespective of the agent used (EPIC, ${ }^{\mathrm{w} 29} \mathrm{EPI}-$

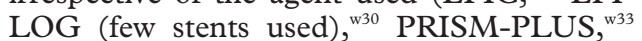
PURSUIT $\left.^{\mathrm{w} 36}\right)$. Debate is ongoing concerning the true benefit of these agents in routine PCI, despite it being advocated by the National Institute for Clinical Evidence, and also whether one agent is "better" than any of the others. The EPISTENT study suggested benefit in stable patients, but it is less clear if the placebo group included patients who would have received this agent normally. However, if there is the belief that the glycoprotein IIb/IIIa inhibitors are to be used in "all PCIs" then two recent trials would support the use of abciximab. The recently presented ESPRIT study (eptifibatide) ${ }^{\mathrm{w} 37}$ indicated benefit only in patients with acute coronary syndrome of $<2$ days' duration $(5.75 \% v 11.1 \%, \mathrm{p}=0.013)$, with no benefit in stable patients $(5.4 \% v 7.2 \%$, NS), and the TARGET study (abciximab $v$ tirofiban) demonstrated superiority of abciximab over tirofiban in all patient groups $(6.01 \%$ v $7.55 \%, \mathrm{p}=0.037)$. ${ }^{\mathrm{3} 3} \mathrm{p}$

There is still the belief, however, that these agents may not be necessary in the routine treatment of patients and the trial to demonstrate this is still needed. While their benefit in those with acute coronary syndrome going onto PCI has been shown, the situation is complicated by the fact that the recent GUSTO IV ACS trial $^{\mathrm{w} 38}$ failed to show a benefit with abciximab in acute coronory syndromes, whereas trials of tirofiban and eptifibatide have.

How a patient presenting with acute coronary syndrome should be treated is unresolved. The evidence suggests that they should be given either tirofiban or eptifibatide (there are unlikely to be any head to head comparisons) but not abciximab, but that if they go on to PCI then abciximab is the preferred agent. There are concerns about both cost and receptor occupancy (and bleeding) if a policy of transferring over from the small synthetic molecules to the antibody is proposed. Most will attempt to triage patients, and transfer and intervene with PCI during the infusion of the small molecule agent (first 72 hours). The debate continues.

\section{Stenting and areas of contention}

\section{Small vessel disease}

Previous trials have suggested high restenosis rates $(>45 \%)$ in those patients with small vessel disease. Four recent studies have produced 


\section{Summary of trials utilising glycoprotein IIb/IIIa inhibitors in patients requiring percutaneous coronary intervention (PCI) Abciximab \\ EPIC Most benefit in subgroup considered} higher risk (acute coronary syndrome) (predominantly balloon angioplasty)

EPILOG Lower dose heparin during the procedure leads to less bleeding with these agents

CAPTURE The benefits, albeit to a lesser degree, occur before PCI in patients with acute coronary syndrome

EPISTENT The use of these agents improves stenting outcome predominantly through a reduction in enzyme defined acute infarction, but additionally there is some mortality benefit especially in diabetics

GUSTO IV ACS Abciximab has no benefits in patients with acute coronary syndrome

\section{Eptifibatide}

PURSUIT Predominantly a study of acute coronary syndrome showing a small absolute benefit $(14.2 \%$ v $15.7 \%)$

IMPACT I and II Predominantly dosing studies

ESPRIT Patients receiving eptifibatide who have acute coronary syndrome do better when undergoing PCI compared to placebo. No benefit was seen in those with stable angina

\section{Tirofiban}

PRISM-PLUS Tirofiban of benefit in those presenting with acute coronary syndrome (probability of death/acute myocardial infarction $0.9 \% \approx 2.6 \%$ ), and especially beneficial in those patients going onto PCI (about a quarter in this study)

RESTORE $98 \%$ of patients with acute coronary syndrome went on to PCI in this study and an early benefit was seen but this was lost by six months, although retained if re-analysed according to the EPOIC data

TARGET Incidence end point reached significantly less with abciximab compared with tirofiban

conflicting results. A number of studies have recently been published. The SISA trial ${ }^{\mathrm{w3}}$ $(n=325)$ showed a trend only towards less adverse outcome with stent (Bestent) versus balloon in vessels $<2.9 \mathrm{~mm}$. This was because the acute gain with stenting $(1.37 \mathrm{~mm} v$ $0.91 \mathrm{~mm}, \mathrm{p}=0.0001$ ) was offset by the greater late loss in the stent group $(28.5 \% v 18.4 \%$, $\mathrm{p}=0.0002)$. In the setting of small vessels such loss cannot be accommodated so easily. The BESMART study ${ }^{\mathrm{w} 40}(\mathrm{n}=381)$ on the other hand (also Bestent) showed a restenosis rate of $22.7 \%$ in the stent group versus $48.8 \%$ in balloon group $(\mathrm{p}<0.0001)$ and a target lesion
The resolved and unresolved problems of stenting

Stents have been shown to be beneficial in: -acute post-angioplasty vessel closure -BENESTENT type lesions (short, simple lesions)

-PCI in coronary vein grafts

-chronic total occlusions

-acute coronary syndromes (unstable

angina and acute myocardial infarction)

- Stent use remains problematic in:

-vessels with reference diameter $<3 \mathrm{~mm}$ (restenosis rates $>25 \%$ compared with approximately $15 \%$ BENESTENT lesions)

-diabetics

-bifurcation disease

- Stent use is as yet unproven in:

-left main stem disease

-preventing restenosis with drug elution

revascularisation rate of $13 \%$ versus $25 \%$, respectively $\quad(p=0.016)$ ISAR-SMART showed no benefit from stenting $(35.7 \% v$ $37.4 \%$, vessel size $2.0-2.8 \mathrm{~mm}$ ) whereas RAP (Garcia) (vessel size 2.2-2.7 mm) demonstrated a restenosis rate $27 \%$ in the stent group versus $37 \%$ in the balloon group. The average restenosis rates for these studies in stents deployed in vessels of between $2.2-2.9 \mathrm{~mm}$ is $27.5 \%$, double that of BENESTENT lesions. It is clear that physicians will wish not to exclude patients from the potential benefit of stenting based on vessel size. It is likely therefore that it is in this group that drug eluting stents may have most impact since intimal hyperplasia will always have greater impact on the smaller lumen.

\section{Left main stem stenting}

It has always been regarded as taboo to undertake PCI on unprotected left main stem disease. However, a number of groups world wide are, through the use of registries, identifying the risk and the patients in whom such intervention could be deemed acceptable. In general early studies such as that by Park suggested excellent results in those at low risk (100\% success rate, $17 \%$ clinical recurrence at six months and only one death), ${ }^{\text {w41 }}$ although Barragan reported three deaths out of 15 high risk patients. ${ }^{\mathrm{w} 42}$ Ellis has reported on the ULTIMA registry and the outcome appeared dependent on patient characteristics, ranging from those with stable angina to those deemed inoperable, as well as left ventricular function status. Two hundred and seventy nine consecutive patients who had left main stem PCI at one of 25 sites between 1993 and 1998 were studied. Forty six per cent of these patients were deemed inoperable or at high surgical risk. Thirty eight patients (13.7\%) died in hospital, and the rest were followed for a mean of 19 months. The one year incidence was $24.2 \%$ for all cause mortality, $20.2 \%$ for 


\section{Trial acronyms}

ACME Angioplasty Compared with Medicine

AMIST Angioplasty versus Minimally Invasive Surgery Trial

ARTS Arterial Revascularization Therapy Study

BARI Bypass Angioplasty Revascularization Investigation

BENESTENT Belgium-Netherlands Stent Study

BESMART Bestent in Small Arteries

CABRI Coronary Angioplasty versus Bypass Revascularisation Investigation

CAPTURE Chimeric 7E3 Anti-Platelet in Unstable Angina Refractory to Standard Treatment

CLASSICS Clopidogrel plus Aspirin Stent International Cooperative Study

EAST Emory Angioplasty versus Surgery Trial

ELUTES Evaluation of Taxol Eluting Stent

EPIC Evaluation of IIb/IIIa platelet receptor antagonist $7 \mathrm{E} 3$ in Preventing Ischemic Complications

EPILOG Evaluation of PTCA to Improve Long-term Outcome by c7E3 GP IIb/IIIa receptor blockade trial

EPISTENT Evaluation of Platelet GP IIb/IIIa Inhibitor for Stenting

EPIC Evaluation of 7E3 for the Prevention of Ischemic Complications

ERACI Argentine Randomized Trial Coronary Angioplasty versus

Coronary Artery Bypass Surgery in Multivessel Disease

ESPRIT European Study of Prevention of Reocclusion after Initial Thrombolysis

FANTASTIC Full Anticoagulation versus Ticlopidine plus Aspirin After Stent Implantation

FINESS First International New Intravascular Rigid-flex Endovascular Stent Study

GABI German Angioplasty Bypass Surgery Investigation

GRAMI GRII stent in Acute Myocardial Infarction

GUSTO Global Use of Strategies To Open Occluded Coronary

Arteries

IMPACT Integrilin to Manage Platelet Aggregation to Combat

Thrombosis Trial

INHIBIT Inhibit Restenosis Intervention with $\beta$-Radiation Trial

ISAR Intracoronary Stenting and Antithrombotic Regimen

ISAR-SMART Intracoronary Stenting or Angioplasty for Restenosis

Reduction in Small Arteries.

MASS The Medicine, Angioplasty or Surgery Study

MUSIC Multicenter Ultrasound Stenting in Coronaries

PRISM-PLUS Platelet Receptor Inhibition in Ischemic Syndrome Management in Patients Limited by Unstable Signs and Symptoms

PURSUIT Platelet IIb/IIIa Underpinning the Receptor for Suppression of Unstable Ischemia Trial

RAVEL Randomized study with sirolimus coated BX Velocity balloon Expandable stent in the treat of patients with de novo native coronary Lesions

REACT Rescue Angioplasty versus Conservative treatment or repeat Thrombolysis

RESTORE Randomized Efficacy Study of Tirofiban for Outcomes and Restenosis

RITA Randomized Intervention Treatment of Angina

SAFER Saphenous vein graft Angioplasty Free of Emboli Randomized trial

SICCO Stenting In Chronic Coronary Occlusion

SISA Stents In Small Arteries

SLIDE Selected Lesion Indication for Direct Stenting

SOS Stent Or Surgery

START Stents and Radiation Therapy Trial

STARS Stent Anticoagulation Regimen Study

STRESS Stent Restenosis Study

TARGET Do Tirofiban And Reopro Give similar Efficacy outcomes Trial

TOSCA Total Occlusion Study of Canada

ULTIMA Unprotected Left main Trunk Intervention Multi-center Assessment.

WEST West European Stent Trial

WIDEST Wiktor Stent in de Novo Stenosis cardiac mortality, 9.8\% for myocardial infarction, and $9.4 \%$ for CABG. Independent correlates of all cause mortality were: left ventricular ejection fraction (LVEF) $\leqslant 30 \%$, mitral regurgitation grade 3 or 4 , presentation with myocardial infarction and shock, creatinine $\geqslant 2.0 \mathrm{mg} / \mathrm{dl}$, and severe lesion calcification. For the $32 \%$ of patients aged $<65$ years, with LVEF $>30 \%$ and without shock, the prevalence of these adverse risk factors was low. No periprocedural deaths were observed in this low risk subset, and the one year mortality was only $3.4 \%$. It is likely that PCI for unprotected left main stem disease will be kept for those at high risk from surgery.

\section{Bifurcation lesions}

The best treatment for bifurcation disease is unresolved. Some would question whether PCI is the treatment of choice, because of technical issues and a high incidence of acute and chronic subsequent events. Stent deployment in both arms of the bifurcation, or the stenting of one and ballooning of the other depending on the presence of disease or the result of intervention, are current topics for debate. While some authors have reported very high restenosis rates, Lefevre ${ }^{\mathrm{w} 43}$ reported major adverse cardiac event rates of $17.1-29.2 \%$ depending on the learning curve. The use of so-called "kissing" balloons appeared to have a beneficial influence on the outcome. Others have shown that stenting of the side branch may not be essential but choosing which to stent and which to leave is the subject of several proposed studies.

\section{Direct stenting}

Direct stenting may have significant advantages over routine procedure where there is predilatation with a balloon first. There appears little doubt that tissue response to the vessel wall is the result of the damage induced by the balloon inflation (and stent deployment). If this damage could be reduced, less tissue response should occur. Additionally the procedure would be cheaper requiring a balloon-stent unit only. There have been a number of reports outlining the feasibility of such a strategy. Hamon recently published the outcome of 122 "carefully selected" patients. Factors such as calcification and tortuosity need to be taken into account when direct stenting is considered. Procedural success was $96 \%{ }^{\text {w }}{ }^{44}$ In five cases it was not possible to deliver the stent through the undilated lesion and the stent was retrieved, but in two cases the stent was lost in the peripheral circulation. The authors rightly reported the need for a controlled trial and one UK study (the SLIDE trial) is underway.

\section{Future directions}

There are three areas of future direction for stents. Firstly, radiation is likely to be incorporated into clinical practice to deal with in-stent restenosis.

The second area of development is the use of stents that carry drugs to inhibit the tissue 
responses. The use of such stents is likely to be cost beneficial because of the small amount of drug required in comparison to systemic treatments, provided the "overall" cost of the stent is not too high and the technology can be applied to longer stents. If shown to be effective in upcoming clinical trials then stents will deal with all four aspects of restenosis; good acute result, recoil, negative remodelling, and instent tissue growth.

Finally, there is likely to be an increase in the combined approach to treating multivessel disease, with the interventionist dealing with lesions in the right coronary and circumflex arteries while the surgeon performs a minimally invasive operation on the left anterior descending artery. The rationale for the combined approach is based on the increased difficulty the surgeon has in using arterial conduits to graft the right and circumflex arteries compared to the left anterior descending artery, which may be best treated with a minimally invasive procedure. If this can be done then angioplasty and stenting of the other diseased vessels rather than using the vein as a conduit may be a good option. Clinical trials of such a combined approach are required.

1. Pocock SJ, Henderson RA, Rickards AF, et al. Meta-analysis of randomised trials comparing coronary angioplasty with bypass surgery. Lancet 1995;346:1184-9 - Important meta-analysis demonstrating the medium term terms of need for revascularisation.

2. Rickards AF, Davies SW. Coronary angioplasty versus coronary surgery in the management of angina. Curr Opin Cardiol 1995;10:399-403

- An overview of balloon angioplasty versus surgery using registry data as well as trial data.

3. Zapolanski A, Rosenblum J, Myler RK, et al. Emergency coronary artery bypass surgery following failed balloon angioplasty: role of the internal mammary artery graft. J Cardiac Surg 1991;6;439-48.

- This study highlighted the risk of acute coronary closure following angioplasty and the failure to use best surgical treatment.

4. Kioka Y, Dallan L, Oliveira S, et al. Clinical experience of emergency coronary artery bypass grafting following faile percutaneous transluminal coronary angioplasty. Jpn J Surg 1991;21:643-9.

- Showed just how high risk failed balloon angioplasty could be.

5. Scott NA, Weintraub WS, Carlin SF, et al. Recent changes in the management and outcome of acute closure after percutaneous transluminal coronary angioplasty. Am J Cardiol 1993;71:1159-63.

- Indicated that there was a problem with balloon angioplasty and set the scene for stenting.

6. Roubin GS, Cannon AD, Aggarwal SK, et al. Intracoronary stenting for acute and threatened closure complicating percutaneous transluminal coronary

angioplasty. Circulation 1992;85:916-27.

- Indicated that stenting may be the answer to acute balloon failure.

7. Schomig A, Kastrati A, Mudra H, et al. Four-year experience with Palmaz-Schatz stenting in coronary angioplasty complicated by dissection with threatened or present vessel closure. Circulation 1994;90:2716-24.

- Important in the development of stenting, suggesting that the treatment of choice for acute problems was the deployment of stents.

8. Mintz GS, Mehran R, Waksman R, et al. Treatment of in-stent restenosis. Seminars in Interventional Cardiology 1998:3:117-21.

- Highlighted the importance of negative remodelling in the development on in-stent restenosis.
9. Kuntz RE, Baim DS. Defining coronary restenosis. Newer clinical and angiographic paradigms. Circulation 1993;88:1310-23.

- A seminal paper indicating that the better the acute gain the more room there was for the development of any in-stent restenosis, irrespective of device used. Stenting was shown to produce and retain the best acute result.

10. Serruys PW, de Jaegere P, Kiemeneij F, et al. A comparison of balloon-expandable-stent implantation with balloon angioplasty in patients with coronary artery disease. Benestent study group. N Engl J Med 1994;331:489-95.

Demonstrated that stenting produced less recurrence than balloon alone, but also that this was caused by the acute benefit. This study also showed that tissue in-growth was greater with stenting.

11. Fischman DL, Leon MB, Baim DS, et al. A randomised comparison of coronary stent placement and balloon angioplasty in the treatment of coronary artery disease. 1994:331:496-501.

- This study also showed the benefits of stenting versus angioplasty, but interestingly the restenosis rates were $10 \%$ higher than in the Benestent trial because of differences in case selection.

12. Dirschinger J, Schuhlen $\mathbf{H}$, Boekstegers $P$, et al Equivalence or difference? One year follow up of a randomised trial of five different slotted-tube stents [abstract] randomised trial of five differ

- Suggested that for whatever reason a stent might be chosen, in general most stents are the same in terms of outcome, providing they are of the same design-that is slotted tube rather than coil tube design.

13. Karsch KR, Newby A. Stent magic! The genie has escaped from the bottle. Heart 2000;84:469-70.

- An important recent commentary expressing the belief, based on the WIDEST study, that there is too much stenting.

14. Serruys PW, Unger F, van Hout BA, et al. The ARTS study (arterial revascularisation therapies study). Seminars in Interventional Cardiology 1999;4:209-19.

- Trial of multivessel stenting versus angioplasty. Previous comparisons had been between balloon angioplasty alone and surgery.

15. Nunes G, Pintto I, Mattos L, et al. Coronary stenting in vessels smaller than $3 \mathrm{~mm}$ is associated with higher restenosis rates [abstract] Eur Heart J 1996;17:173.

- Highlighted the problems when there is insufficient room, as with small reference vessels, for the intimal hyperplasia to be accomodated.

16. Ponde CK, Watson PS, Aroney CN, et al. Multiple stent implantation in single coronary arteries: acute results and six month angiographic follow up. Cathet Cardiovasc Diag 1997;42:158-65.

- Found higher risk of restenosis in patients receiving multiple stents, but reason unknown.

17. Lau KW, Ding ZP, Johan A, et al. Midterm angiographic outcome of single-vessel intracoronary stent placement in diabetic versus non-diabetic patients: a matched

comparative study. Am Heart J 1998;136:150-5.

- Diabetics remain at high risk of in-stent restenosis, especially in small vessel reference diameter.

18. Popma J, Heuser R, Suntharalingam M, et al for the START Investigators. Late clinical and angiographic outcomes after use of ${ }^{90} \mathrm{Sr} /{ }^{90} \mathrm{Y}$ beta radiation for the treatment of in-stent restenosis. Results from the stents and radiation therapy (START) trial [abstract]. J Am Coll Cardiol 2000;35.

- Definitive study demonstrating the benefit of $\beta$ emitter radiation for treatment of in-stent restenosis.

19. Waksman $\mathbf{R}$, Raizner A, Lansky A, et al. Beta radiation to inhibit recurrence of in-stent restenosis: study design, device and dosimetry details of the multicenter randomised double blind study [abstract]. Circulation

- A second trial showing benefit of a $\beta$ emitter (32 P) in in-stent restenosis compared to balloon angioplasty alone.

20. Sirnes PA, Golf S, Myreng Y, et al. Stenting in chronic coronary occlusion (SICCO): a randomised controlled trial of adding a stent implantation after successful angioplasty. $J$ Am Coll Cardiol 1996;28:1444-51.

- An important study which suggested that in the short term at least those patients with chronic occlusion were better off receiving a stent than just being treated with balloon angioplasty alone.

21. Windecker S, Meier B. Intervention in coronary artery disease. Heart 2000;83:481-90.

- Previous article in the Education in Heart series addressing adjunctive pharmacological treatment in PCI. website

extra

Additional references appear on the Heart website

www.heartjnl.com 